\title{
Analysis of Two-Echelon Perishable Inventory System with Direct and Retrial demands
}

\author{
M. Rameshpandi ${ }^{1}$ C.Periyasamy ${ }^{2} \quad$ K. Krishnan ${ }^{3}$ \\ ${ }^{I}$ Department of Mathematics, P. M. Thevar College, Usilampatti, Madurai Dt.,Tamil Nadu, India. \\ ${ }^{2}$ Department of Mathematics, Madurai Kamaraj University College, Sattur, Virudhunagar Dt.,Tamil Nadu, \\ India. \\ ${ }^{3}$ Research Department of Mathematics, C.P.A College, Bodinayakanur, Theni Dt., Tamil Nadu, India.
}

\begin{abstract}
In this paper, we consider a continuous review perishable inventory system with Markovian demand. The operating policies are $(s, S)$ and $(0, M)$. The life time of an item has an exponential distribution. The ordered items are received after a random time which is assumed to be exponential distribution. The demands that occur directly to the distribution centre are called direct demands. The arrival process for the direct demand follows Poisson process. The demand process to the retailer node is independent to the direct demand process and follows Poisson process. The demands that occur during stock out period are enter into the orbit of finite size. These orbiting demands retry for their demand after a random time, which is assumed to be exponential distribution. The joint probability distribution of the inventory level at lower echelon, higher echelon and the number of customer in the orbit is obtained in the steady state case. Various system performance measures in the steady state are derived and the long run total expected cost rate is calculated.
\end{abstract}

Keywords: Two-echelon Inventory system, Perishable Inventory, Retrial demand, Direct demand.

\section{Introduction}

A supply chain may be defined as an integrated process wherein a number of various business entities (suppliers, distributors and retailers) work together in an effort to (1) acquire raw materials (2) process them and then produce valuable products and (3) transport these final product to retailers. The process and delivery of goods through this network needs efficient communication and transportation system. The supply chain is traditionally characterized by a forward flow of materials and products and backward flow of information. Over the last two decades, researchers and practitioners have primarily investigated the various process of supply chain individually.

A Complete review was provided by Benita M. Beamon (1998)[10]. Recently however, there has been increasing attention placed on performance, design and analysis of the supply chain as a whole. HP's(Hawlett Packard) Strategic Planning and Modeling(SPaM) group initiated this kind of research in 1977. From practical stand point the supply chain concept arose from a number of changes in the manufacturing environment, including the rising costs of manufacturing, the shrinking resources of manufacturing bases, shortened product life cycles, the levelling of planning field within manufacturing, inventory driven costs (IDC) involved in distribution and the globalization of market economics. With in manufacturing research, the supply chain concept grow largely out of two-stage multi-echelon inventory models, and it is important to note that considerable research in this area is based on the classic work of Clark and Scarf (1960)[1].

A complete review on this development were recorded by Federgruen (1993)[9]. Recent developments in two-echelon models may be found in Q. M. He, and E. M. Jewkes (2000)[11], Axsater.S (1993)[7] and Nahimas, S(1982)[4]. Continuous review Perishable inventory with instantaneous replenishment was considered by Kalpakam, S and Arivarignan, G(1988)[5]. A continuous review ( $\mathrm{s}, \mathrm{S}$ ) policy with positive lead times in two echelon Supply Chain was considered by Krishnan.K and Elango.C. 2005 [15][16].

This paper deals with a simple supply chain that is modelled as a single warehouse and multiple retailer system handling a single product. In order to avoid the complexity, at the same time without loss of generality, we assumed identical demand pattern at each node. This restricts our study to design and analyze a tandem network of inventory, which is a building block for the whole supply chain system. The rest of the paper is organized as follows. The model formulation is described in section 2, along with some important notations used in the paper. In section 3, steady state analysis are done. Section 4 deals with the operating characteristics of the system and section 5 deals with the cost analysis for the operation. The section 6 deals with some numerical illustration and the last section 7 concludes the paper. 


\section{Model Description}

In this paper, we consider a two level supply chain inventory system. It consists of one warehousing facility and one retailer. We assumed that the demands to the Distribution Centre follow Poisson process with parameter $\lambda_{\mathrm{D}}(>0)$. The direct demand gets $\mathrm{Q}$ units at a time. The demands initiated at retailer node follow Poisson process with parameter $\lambda_{\mathrm{P}}(>0)$. The demand to the retailer node requires single item at a time. The lead times are exponentially distributed with parameter $\mu(>0)$. The retailer follows $(\mathrm{s}, \mathrm{S})$ policy to maintain inventory and the distributor follow $(0, n Q)$ policy for maintaining inventory. The items are perishable in nature, and it is assumed that the items are perishes only at the retailer node. The life time of an item is exponentially distributed with parameter $\gamma(>0)$. The unsatisfied customers are treated as retrial customers and they are waiting in the orbit with finite capacity N. The repeated customers from the orbit (with capacity i) are entered into the system with rate $\mathrm{i} \lambda_{\mathrm{R}}(>0)$. The arriving demands finds the empty stock and the orbit is full are considered to be lost.

\section{Notation:}

- $[A]_{i, j}:(\mathrm{i}, \mathrm{j})$ th element/block of the matrix A

- $I_{n}$ : Identity matrix of order $\mathrm{n}$

- $\quad e$ : column vector of ones with appropriate dimension

- $\quad \mathrm{S}=$ The maximum inventory level at retailer nodes

- $\quad \mathrm{s}=$ Reorder level at retailer nodes

- $\mathrm{Q}=\mathrm{S}-\mathrm{s}$

- $\mathrm{E}=\{(i, j, k) \mid i=0,1, \ldots, N, j=0,1, \ldots, S, k=Q, 2 Q, \ldots, n Q\}$

\section{Analysis}

Let $\left\{\mathrm{T}_{1}(\mathrm{t}), \mathrm{T}_{2}(\mathrm{t}), \mathrm{T}_{3}(\mathrm{t}): \mathrm{t} \geq 0\right\}$ respectively denote the number of demands in the orbit, the on hand inventory level in the retailer node and the number of items in the Distribution centre at time t. From the assumptions made on the input and output processes $X(t)=\left\{\left(T_{1}(t), T_{2}(t), T_{3}(t)\right): t \geq 0\right\}$ is a Markov process with state space $\mathrm{E}$. The infinitesimal generator of this process $\mathrm{A}=(\mathrm{a}(\mathrm{i}, \mathrm{k}, \mathrm{m}: \mathrm{j}, \mathrm{l}, \mathrm{n})),(\mathrm{i}, \mathrm{k}, \mathrm{m}),(\mathrm{j}, \mathrm{l}, \mathrm{n}) \in \mathrm{E}$ can be obtained from the following arguments.

- The primary arrival of demand to the retailer node makes a transition in the Markov process from ( $\mathrm{i}, \mathrm{k}$, $\mathrm{m})$ to $(\mathrm{i}, \mathrm{k}-1, \mathrm{~m})$ with intensity of transition $\lambda_{\mathrm{P}}$.

- The arrival of a demand at retailer node from orbit makes a transition in the Markov Process from (i, $\mathrm{k}, \mathrm{m})$ to $(\mathrm{i}-1, \mathrm{k}-1, \mathrm{~m})$ with intensity of transition $\lambda_{\mathrm{R}}$.

- The item perishes makes the transition from (i, $\mathrm{k}, \mathrm{m})$ to $(\mathrm{i}, \mathrm{k}-1, \mathrm{~m})$ with intensity of transition $\mathrm{k} \gamma$.

- The replenishment of an inventory at retailer node makes a transition from $\quad(i, k, m)$ to $(i, k+Q, m-$ Q) with rate of transition $\mu$.

- The arrival of demand to the distribution centre makes a transition in the Markov process from (i, $\mathrm{k}$, $\mathrm{m})$ to $(\mathrm{i}, \mathrm{k}, \mathrm{m}-\mathrm{Q})$ with intensity of transition $\lambda_{\mathrm{D}}$.

Then, the infinitesimal generator has the following finite QBD structure:

$$
P=\begin{aligned}
& 0 \\
& 0 \\
& 1 \\
& 2 \\
& \vdots \\
& N-2 \\
& N-1 \\
& N
\end{aligned}\left(\begin{array}{ccccccc}
A_{0} & C & & & & & \\
B_{1} & A_{1} & C & & & & \\
& B_{2} & A_{2} & & & & \\
& & \ddots & \ddots & \ddots & & \\
& & & & A_{N-2} & C & \\
& & & & B_{N-1} & A_{N-1} & C \\
& & & & & B_{N} & A_{N}
\end{array}\right)
$$

Where,

$$
[C]_{i, j}=\left\{\begin{array}{cc}
\lambda_{P} I_{n}, & j=i \quad i=0 \\
0 & \text { otherwise }
\end{array}\right.
$$

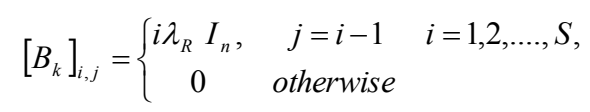

$$
k=0,1,2, \ldots . ., N
$$




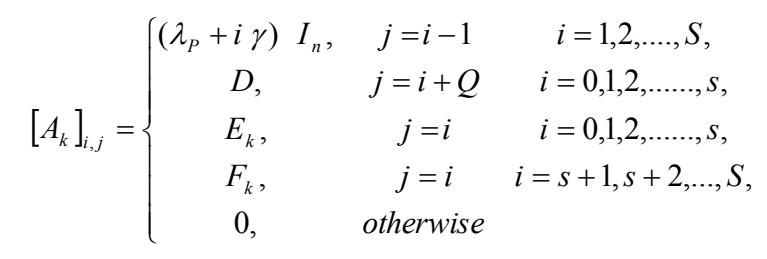

where

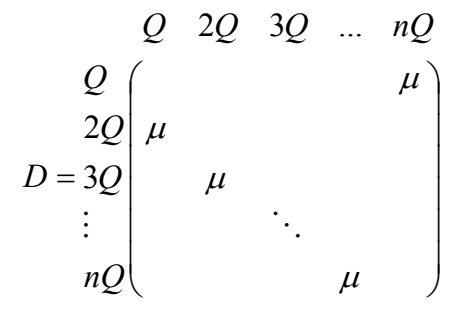

$$
\begin{aligned}
& \begin{array}{lllll}
Q & 2 Q & 3 Q & \ldots & n Q
\end{array}
\end{aligned}
$$

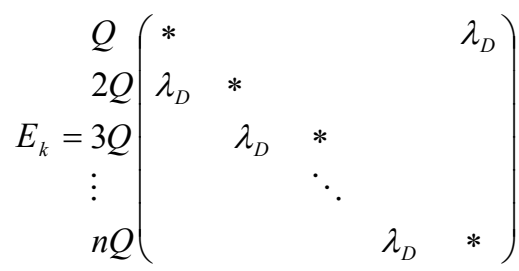

Where $*=-\left(\lambda_{D}+\lambda_{P}+i \gamma+k \lambda_{R}+\mu\right)$

$$
\begin{aligned}
& \begin{array}{lllll}
Q & 2 Q & 3 Q & \ldots & n Q
\end{array} \\
& \begin{aligned}
& Q \\
{\left[E_{N}\right]_{0,0}=} & 2 Q \\
& 3 Q \\
& \\
& n Q
\end{aligned}\left(\begin{array}{cccccc}
\Delta & & & & \lambda_{D} \\
\lambda_{D} & \Delta & & & \\
& \lambda_{D} & \Delta & & \\
& & \ddots & & \\
& & & \lambda_{D} & \Delta
\end{array}\right)
\end{aligned}
$$

Where $\Delta=-\left(\lambda_{D}+k \lambda_{R}+\mu\right)$

$$
\begin{aligned}
& \begin{array}{lllll}
Q & 2 Q & 3 Q & \ldots & n Q
\end{array} \\
& {\left[E_{N}\right]_{i, j}=\begin{array}{l}
Q Q \\
2 Q \\
\\
\vdots \\
\\
n Q
\end{array}\left(\begin{array}{llllll}
* & & & & \lambda_{D} \\
\lambda_{D} & * & & & \\
& \lambda_{D} & * & & \\
& & \ddots & & \\
& & & \lambda_{D} & *
\end{array}\right)}
\end{aligned}
$$

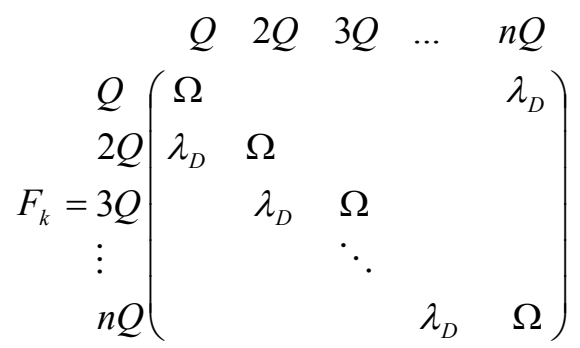

$$
\begin{aligned}
& \text { Where } \Omega=-\left(\lambda_{D}+\lambda_{P}+i \gamma+k \lambda_{R}\right), \quad k=0,1,2, \ldots ., N, \quad i=1,2,3, \ldots . ., S
\end{aligned}
$$

Here the matrices $A_{k}, B_{k}$ and $C$ are the square matrices of order $(S+1)^{*} n$ and note that the matrix $D$ is the square matrix of order $n$. 


\subsection{Steady State Analysis}

Since the state space is finite and $\mathrm{P}$ is irreducible, the stationary probability vector $\Pi$ for the generator $\mathrm{P}$ always exists and satisfies

The vector $\Pi$ can be represented by

$$
\Pi P=0 \& \Pi e=1 .
$$

$$
\begin{aligned}
& \Pi=\left(\Pi^{<0>}, \Pi^{<1>}, \Pi^{<2>}, \ldots \ldots, \Pi^{<N>}\right) \\
& \Pi^{\langle i\rangle}=\left(\pi^{\langle i, 0\rangle\rangle}, \pi^{\langle i, 1,>\rangle}, \ldots \ldots, \pi^{\langle<i, S>\rangle}\right) \\
& \Pi^{\langle<i, j\rangle\rangle}=\left(\pi^{\langle\langle i, j, Q\rangle\rangle}, \pi^{\langle\langle i, j, 2 Q\rangle \gg\rangle}, \ldots \ldots ., \pi^{\langle\langle i, j, n Q\rangle \gg\rangle}\right), \quad j=0,1,2, \ldots . ., S .
\end{aligned}
$$

Now the structure of $\mathbf{P}$ shows, the model under study is a finite birth death model in the Markovian environment. Hence we use the Gaver algorithm for computing the limiting probability vector. For the sake of completeness we provide the algorithm here.

\section{Algorithm:}

1. Determine recursively the matrix $D_{n}$,

$$
0 \leq \mathrm{n} \leq \mathrm{N} \text { by using } \mathrm{D}_{0}=A_{0} \quad \mathrm{D}_{\mathrm{n}}=A_{n}+B_{n}\left(-D_{n-1}^{-1}\right) C, . \quad n=1,2, \ldots . K
$$

2. Solve the system $\Pi^{<N>} D_{N}=0$.

3. Compute recursively the vector

$$
\begin{gathered}
\Pi^{<n>}, \quad n=N-1, \ldots ., 0 \text { using } \\
\Pi^{<\mathrm{n}>}=\Pi^{<n+1>} B_{n+1}\left(-D_{n}^{-1}\right), n=n-1, \ldots \ldots, 0 .
\end{gathered}
$$

4. Re-normalize the vector $\Pi$, using

$$
\Pi e=1 \text {. }
$$

\section{Performance Measures}

In this section we computed the following system performance measures in steady for proposed inventory system

\subsection{Mean Inventory Level}

Let $I_{R}$ denote the expected inventory level in the steady state at retailer node and $I_{D}$ denote the expected inventory level at distribution centre.

$$
\mathrm{I}_{\mathrm{R}}=\sum_{i=0}^{N} \sum_{j=1}^{S} j \pi^{<i, j>>} e \quad \mathrm{I}_{\mathrm{D}}=\sum_{i=0}^{N} \sum_{j=0}^{S} \sum_{k=Q}^{n Q} k \pi^{<<i, j, k>\gg>}
$$

\subsection{Expected Perishable Rate}

Let $I_{P}$ denote the expected perishable rate and which is given by

$$
\mathrm{I}_{\mathrm{P}}=\sum_{i=0}^{N} \sum_{j=1}^{S} j \gamma \pi^{<<i, j>>} e
$$

\subsection{Expected Number Of Demands In The Orbit}

Let $\mathrm{E}(\mathrm{o})$ denote the expected number of customer in the orbit which is given by

\subsection{Mean Reorder Rate}

$$
\mathrm{E}(\mathrm{o})=\sum_{i=1}^{N} i \pi^{<i>} e
$$

The mean reorder rate at retailer node is given by

\subsection{Shortage Rate}

$$
\mathrm{r}_{\mathrm{R}}=\sum_{i=0}^{N}\left(\lambda_{P}+i \lambda_{R}+(s+1) \gamma\right) \pi^{<i, s+1>>} e
$$
given by

Shortage occurs only at retailer node and the shortage rate for the retailer is denoted by $\alpha_{\mathrm{R}}$ and which is 


$$
\alpha_{\mathrm{R}}=\sum_{i=0}^{N} \sum_{k=Q}^{n Q} \lambda_{P} \pi^{<<<i, 0, k>\gg>}+\sum_{i=1}^{N} \sum_{k=Q}^{n Q} i \lambda_{R} \pi^{<<i, 0, k>\gg>}
$$

\section{Cost Analysis}

In this section we analyse the cost structure for the proposed models by considering the minimization of the steady state total expected cost per time.

The long run expected cost rate for the model is defined to be

$$
T C(S, s, n)=h_{R} I_{R}+h_{D} I_{D}+k_{R} r_{R}+g_{R} \alpha_{R}+I_{P} p c+c_{0} E(o)
$$

Where

$\mathrm{h}_{\mathrm{R}^{-}}$denotes the inventory holding cost at retailer node per unit item

$\mathrm{h}_{\mathrm{D}^{-}}$denotes the inventory carrying cost at distribution centre per unit item

$\mathrm{k}_{\mathrm{R}^{-}}$denotes the setup cost/ order at retailer node

$\mathrm{g}_{\mathrm{R}^{-}}$- denotes the shortage cost/ unit shortage at retailer node

$\mathrm{c}_{\mathrm{o}^{-}}$denotes the back ordering of a demand in the orbit / unit time.

pc - denotes cost per unit failure

\section{Numerical Illustration}

We fix the following inputs to calculate the system performance measures and the total inventory cost incurred in all the three nodes

We analysed the following in the numerical section.

1. From Figure 1, we have shown that TC decreases when $\mu, \gamma$ increases.

2. From Table 1 to table 4, we observe the following

a) As is to be expected $\mathrm{s}$ and $\mathrm{S}$ increase, TC increases

b) As is to be expected $\mathrm{N}$ increases, $\mathrm{TC}$ decreases.

c) As is to be expected the total cost function increases when the costs, set-up cost, holding cost, shortage cost, cost for failure and the back ordering cost increase.

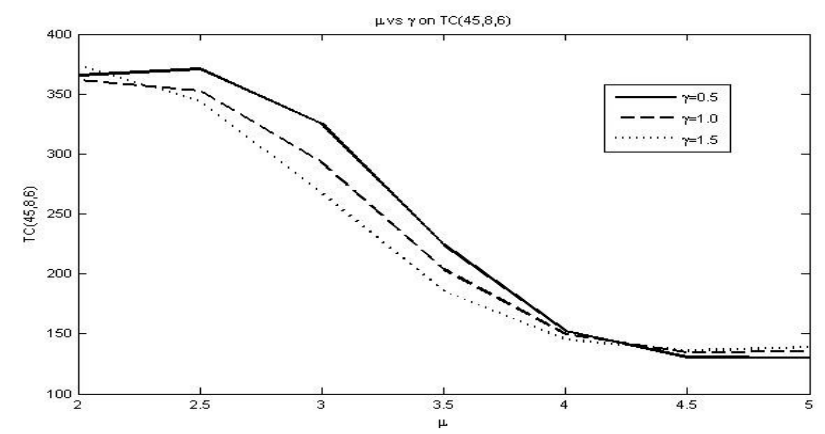

Figure1: $\mu$ vs $\gamma$ on TC $(24,4,6)$

\begin{tabular}{|l|l|l|l|l|l|}
\hline & $\mathbf{s}=\mathbf{2}$ & $\mathbf{s}=\mathbf{4}$ & $\mathbf{s}=\mathbf{6}$ & $\mathbf{s}=\mathbf{8}$ & $\mathbf{s}=\mathbf{1 0}$ \\
\hline $\mathbf{S}=\mathbf{4 5}$ & 111.998164 & 118.761642 & 124.799813 & 130.664478 & 136.843108 \\
\hline $\mathbf{S}=\mathbf{5 0}$ & 118.699318 & 124.359054 & 129.299781 & 133.996082 & 138.773110 \\
\hline $\mathbf{S}=\mathbf{5 5}$ & 123.090318 & 127.562168 & 131.434202 & 135.090008 & 138.765317 \\
\hline $\mathbf{S}=\mathbf{6 0}$ & 125.576395 & 129.128145 & 132.243072 & 135.192545 & 138.137225 \\
\hline $\mathbf{S}=\mathbf{6 5}$ & 126.984419 & 130.065937 & 132.851239 & 135.511516 & 138.145444 \\
\hline
\end{tabular}

Table 1: s vs S on TC

\begin{tabular}{|l|l|l|l|l|l|}
\hline & $\mathbf{N}=\mathbf{8 0}$ & $\mathbf{N}=\mathbf{8 5}$ & $\mathbf{N}=\mathbf{9 0}$ & $\mathbf{N}=\mathbf{9 5}$ & $\mathbf{N}=\mathbf{1 0 0}$ \\
\hline $\mathbf{S}=\mathbf{5 1}$ & 141.797324 & 133.901408 & 123.701280 & 113.740410 & 105.466382 \\
\hline $\mathbf{S}=\mathbf{5 2}$ & 145.639486 & 139.011501 & 129.397952 & 119.220110 & 110.421739 \\
\hline $\mathbf{S}=\mathbf{5 3}$ & 149.044251 & 143.722821 & 134.966776 & 124.794747 & 115.506160 \\
\hline $\mathbf{S}=\mathbf{5 4}$ & 152.064630 & 147.994707 & 140.313344 & 130.414851 & 120.733658 \\
\hline $\mathbf{S}=\mathbf{5 5}$ & 154.768122 & 151.818960 & 145.349846 & 136.007655 & 126.099004 \\
\hline
\end{tabular}

Table 2: $\mathbf{S}$ vs $\mathbf{N}$ on $\mathbf{T C}$ 
Analysis of Two-Echelon Perishable Inventory System with Direct and Retrial demands

\begin{tabular}{|l|l|l|l|l|l|}
\hline & $\mathbf{h}_{\mathbf{D}}=\mathbf{0 . 0 4}$ & $\mathbf{h}_{\mathbf{D}}=\mathbf{0 . 0 8}$ & $\mathbf{h}_{\mathbf{D}}=\mathbf{0 . 1 2}$ & $\mathbf{h}_{\mathbf{D}}=\mathbf{0 . 1 6}$ & $\mathbf{h}_{\mathbf{D}}=\mathbf{0 . 2 0}$ \\
\hline $\mathbf{h}_{\mathbf{R}}=\mathbf{0 . 0 0 2 0 0 0}$ & 29.953186 & 30.429272 & 30.905357 & 31.381443 & 31.857528 \\
\hline $\mathbf{h}_{\mathbf{R}}=\mathbf{0 . 0 0 4 0 0 0}$ & 29.960973 & 30.437059 & 30.913144 & 31.389230 & 31.865315 \\
\hline $\mathbf{h}_{\mathbf{R}}=\mathbf{0 . 0 0 6 0 0 0}$ & 29.968760 & 30.444845 & 30.920931 & 31.397016 & 31.873102 \\
\hline $\mathbf{h}_{\mathbf{R}}=\mathbf{0 . 0 0 8 0 0 0}$ & 29.976546 & 30.452632 & 30.928718 & 31.404803 & 31.880889 \\
\hline $\mathbf{h}_{\mathbf{R}}=\mathbf{0 . 0 1 0 0 0 0}$ & 29.984333 & 30.460419 & 30.936504 & 31.412590 & 31.888675 \\
\hline
\end{tabular}

Table 3: $h_{R}$ vs $h_{D}$ on $T C(24,4,6)$

\begin{tabular}{|l|l|l|l|l|l|}
\hline & $\mathbf{g}_{\mathbf{R}}=\mathbf{0 . 2}$ & $\mathbf{g}_{\mathbf{R}}=\mathbf{0 . 4}$ & $\mathbf{g}_{\mathbf{R}}=\mathbf{0 . 6}$ & $\mathbf{g}_{\mathbf{R}}=\mathbf{0 . 8}$ & $\mathbf{g}_{\mathbf{R}}=\mathbf{1 . 0}$ \\
\hline $\mathbf{k}_{\mathbf{D}}=\mathbf{1 0}$ & 23.104477 & 23.619900 & 24.135323 & 24.650745 & 25.166168 \\
\hline $\mathbf{k}_{\mathbf{D}}=\mathbf{1 5}$ & 24.431902 & 24.947325 & 25.462748 & 25.978170 & 26.493593 \\
\hline $\mathbf{k}_{\mathbf{D}}=\mathbf{2 0}$ & 25.759327 & 26.274750 & 26.790173 & 27.305595 & 27.821018 \\
\hline $\mathbf{k}_{\mathbf{D}}=\mathbf{2 5}$ & 27.086752 & 27.602175 & 28.117598 & 28.633021 & 29.148443 \\
\hline $\mathbf{k}_{\mathbf{D}}=\mathbf{3 0}$ & 28.414177 & 28.929600 & 29.445023 & 29.960446 & 30.475868 \\
\hline
\end{tabular}

Table 4: $k_{D}$ vs $g_{R}$ on TC $(24,4,6)$

\begin{tabular}{|l|l|l|l|l|l|}
\hline & $\mathbf{p c}=\mathbf{0 . 2}$ & $\mathbf{p c}=\mathbf{0 . 4}$ & $\mathbf{p c}=\mathbf{0 . 6}$ & $\mathbf{p c}=\mathbf{0 . 8}$ & $\mathbf{p c}=\mathbf{1 . 0}$ \\
\hline $\mathbf{c}_{\mathbf{0}}=\mathbf{2}$ & 15.749604 & 16.250335 & 16.751066 & 17.251797 & 17.752528 \\
\hline $\mathbf{c}_{\mathbf{0}}=\mathbf{3}$ & 18.257545 & 18.758275 & 19.259006 & 19.759737 & 20.260468 \\
\hline $\mathbf{c}_{\mathbf{0}}=\mathbf{4}$ & 20.765485 & 21.266216 & 21.766946 & 22.267677 & 22.768408 \\
\hline $\mathbf{c}_{\mathbf{0}}=\mathbf{5}$ & 23.273425 & 23.774156 & 24.274887 & 24.775617 & 25.276348 \\
\hline $\mathbf{c}_{\mathbf{0}}=\mathbf{6}$ & 25.781365 & 26.282096 & 26.782827 & 27.283558 & 27.784288 \\
\hline
\end{tabular}

Table 5: pc vs c on TC(24, 4, 6)

\section{Concluding Remarks}

In this paper we analysed a continuous review perishable inventory system with supply chain. The structure of the chain allows vertical movement of goods from distribution center to retailers. A (s, Q) type inventory system with Poisson demand and exponential distributed lead times for items are assumed at DC (middle echelon). Demands occurring during the stock out periods are assumed to be lost. The DC replenishes their stocks with exponential distributed lead times from warehouse (upper echelon) has abundant supply source. The items are supplied to the DC in packs of Q (= S-s) items from the warehouse. The model deals with lost sales at DC and the supply from manufacturer is in terms of pockets. It would be interesting to analyse the problem discussed in this article where the life time of items are constant. Naturally, with the inclusion of constant life time of each items, the problem will be more challenging. Another important extension could be made by relaxing the assumption of exponentially distributed lead times to a class of arbitrarily distributed lead times using techniques from renewal theory and semi-regenerative processes. Once this is done, the general model can be used to generate various special cases. For example, three different lead time distributions one with coefficient of variation greater than one, one with coefficient of variation less than one and another with coefficient of variation equal to one can be compared. Cost analysis can then be carried out for (s, Q), (S, S-1) and lot-for-lot models using each of the three different lead time distributions to determine which policy is optimal for any given lead time distribution. The author is currently working on the above extensions, and these will be reported in future publications.

\section{References}

[1] Clark, A. J. and H. Scarf, 1960. Optimal Policies for a Multi- Echelon Inventory Problem. Management Science, 6(4): 475-490.

[2] Hadley, G and Whitin, T. M., (1963), Analysis of inventory systems, Prentice- Hall, Englewood Cliff, New Jersey.

[3] Cinlar, E. Introduction to Stochastic Processes. Prentice Hall, Englewood Cliffs, NJ, 1975.

[4] Nahimas, S. 1982. Perishable inventory theory. A review. Operations Research, 30, 680-708

[5] Kalpakam, S and Arivarignan, G. 1988. A Continuous review Perishable Inventory Model, Statistics 19, 3, 389-398.

[6] Svoronos, Antony and Paul Zipkin, 1991. Evaluation of One-for-One Replenishment Policies for Multiechelon Inventory Systems, Management Science, 37(1): 68-83.

[7] Axsater, S. 1993a. Exact and approximate evaluation of batch ordering policies for two level inventory systems. Oper. Res. 41. 777-785.

[8] Buzacott, J. A. and J. G. Shanthikumar. 1993. Stochastic Models of Manufacturing Systems. Prentice Hall, NJ

[9] Federgruen. A. 1993. Centralized planning models for multi echelon inventory system under uncertainty, S.C.Graves et al.eds. Handbooks in ORMSvol4,North-Holland, Amsterdam, The Netherlands, 133-173.

[10] Benita M. Beamon. 1998. Supply Chain Design and Analysis: Models and Methods. International Journal of Production Economics.Vol.55, No.3, pp.281 294.

[11] Qi. Ming He and E. M. Jewkes. 2000. Performance measures of a make-to- order inventory- production system. IIE Transactions, 32, 409-41

[12] Elango, C., 2001, A continuous review perishable inventory system at service facilities, unpublished Ph. D., Thesis, Madurai Kamaraj University, Madurai

[13] Arivarignan, G., Elango, C. and Arumugam, N., (2002), A continuous review perishable inventory control system at service facilities, Advances in Stochastic Modelling, Notable Publications, Inc., 29 - 40.

[14] Gianpaolo Callioni, Xavier de Montgros et. al. 2005. Inventory Driven Cost, Harvard Business Review, 135-141. 
[15] Krishnan. K and Elango.C. 2005. A continuous review (s, S) policy with positive lead times in Supply Chain, Proceedings of National Conference on Intelligent Optimization Modeling. Allied Publishers Pvt. Itd., New Delhi, 245-253.2005

[16] Krishnan. K, 2007, Stochastic Modeling In Supply Chain Management System, unpublished

Ph.D., Madurai Kamaraj University, Madurai.

Thesis, 